\section{Thinnest generator of electricity}

single layer of molybdenum disulAfide $\left(\mathrm{MoS}_{2}\right), 0.6 \mathrm{~nm}$ thick, generates a peak electrical output of $15 \mathrm{mV}$ and 20 pA when strained by $0.53 \%$, which corresponds to a mechanical-to-electrical energy conversion of $5.8 \%$. These observations were made by a research team from Georgia Institute of Technology (Georgia Tech) and Columbia University, who published their results in the October 15 online edition of Nature (DOI:10.1038/ nature13792).

The accumulation of electric charge in a material when it is stressed is known as piezoelectricity, a phenomenon discovered in 1880 by the brothers Pierre and Jacque Curie. $\mathrm{MoS}_{2}$ (molybdenite in its mineral form) was studied as a source of molybdenum by Scheele in 1778 . However, this material in bulk form cannot be piezoelectric because it has a center of symmetry, and charges from the layers cancel each other out. But in the form of a monolayer - that is a single atomic layer of Mo sandwiched between two adjacent atomic layers of S packed in a hexagonal lattice-it is no longer centrosymmetric and hence becomes piezoelectric. Theoretical work has suggested this possibility for many transition-metal dichalcogenides. Karel-Alexander Duerloo, Mitchell Ong, and Evan Reed at Stanford University calculated the piezoelectric coefficient of the $\mathrm{MoS}_{2}$ monolayer to be $364 \mathrm{pC} / \mathrm{m}$, using density functional theory. A research team from the University of California-Berkeley and Lawrence Berkeley National Laboratory posted a value of $290 \mathrm{pC} / \mathrm{m}$ on the arxiv. org website on August 29, 2014, from inverse piezoelectric measurements (i.e. apply voltage and measure change in force).

Electronics fabricated using piezopotential as a gate voltage to tune or control the charge-transport behavior across a metal/semiconductor interface or a $p-n$ junction are called piezotronics. Transport measurements show for the first time a strong piezotronic effect in single-layer $\mathrm{MoS}_{2}$, but not in bilayer and bulk $\mathrm{MoS}_{2}$. According to Zhong Lin Wang of Georgia Tech, a corresponding author of the article in Nature, "Proof of the piezoelectric effect and piezotronic effect adds new functionalities to these two-dimensional materials." These properties greatly expand the application of layered materials for human-machine interfacing, robotics, microelectromechanical systems, and active flexible electronics.

James Hone, whose team at Columbia prepared the $\mathrm{MoS}_{2}$ flakes and determined their crystal orientations, says, "This material - just a single layer of atoms - could be made as a wearable device, perhaps integrated into clothing, to convert energy from your body movement to electricity and power wearable sensors or medical devices, or perhaps to supply enough energy to charge your cell phone in your pocket."

The study demonstrates that the piezoelectric output can be controlled according to the number of $\mathrm{MoS}_{2}$ atomic layers ( $\mathrm{n})$. When $\mathrm{n}$ is even the material

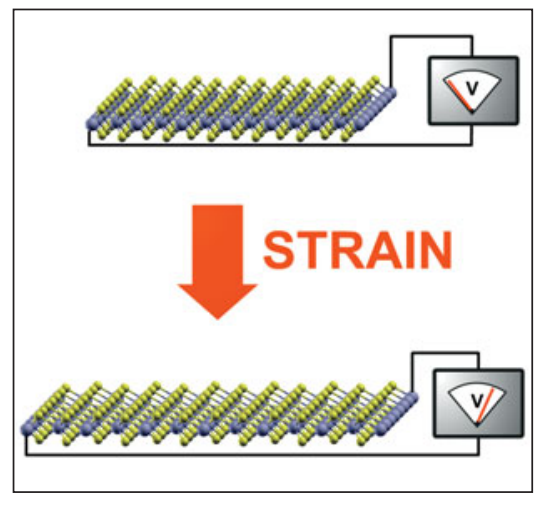

Piezoelectricity in a monolayer of $\mathrm{MoS}_{2}$. The larger atom is Mo. Credit: Evan Reed at Stanford University.

is centrosymmetric and does not show a piezoelectric response. An odd number of layers gives rise to a piezoelectric output, where this is large and decreases roughly as the inverse of $n$. It also shows that serial connection of individual single-layer $\mathrm{MoS}_{2}$ crystals flakes in a circuit can be used to boost the piezoelectric output for energy conversion.

Reed, Duerloo, and Ong, who have studied monolayers and bilayers of twodimensional solids, extensively describe Wang and Hone's work as creative because it has looked at the problem from many angles, and has rigorously characterized the material, which should bring it closer to device fabrication.

They point out that there are many other two-dimensional materials whose monolayer properties may turn out to be superior to those of currently used materials.

N. Balasubramanian
Digital metamaterial bits for simpler optical elements

$I^{2}$ $\mathrm{n}$ this Information Age, digital electronics have become a crucial part of our everyday lives. Binary, or Boolean, logic has become ubiquitous in a society so closely affiliated with personal electronics. The deceptively simple nature of the mathematical structure that uses $1 \mathrm{~s}$ and $0 \mathrm{~s}$ has enabled applications across a range of varied scientific fields.

In the September 14 issue of Nature Materials (DOI: 10.1038/NMAT4082), researchers at the University of Pennsylvania have proposed new methods of producing optical system designs using digital metamaterial "bits" and "bytes." Using simulations constructed with the COMSOL Multiphysics software, the researchers simulated the effective permittivity of two-component structures they label as metamaterial bytes; these were generated in both two-dimensional (2D) rectangular and concentric core-shell configurations. Each byte consisted of two bits, here comprising $\mathrm{Ag}$ and $\mathrm{SiO}_{2}$. It was seen that by altering factors such as bit order, relative bit size, and orientation of the incident wave's electric-field polarization, significant changes in the effective permittivity of the byte could be achieved. Additionally, permittivity values could 